\title{
Description of a stochastic system by a nonadapted stochastic process
}

\author{
Piero Olla* \\ ISAC-CNR and INFN, Sez. Cagliari, I-09042 Monserrato, Italy.
}

\begin{abstract}
An approach for the description of stochastic systems is derived. Some of the variables in the system are studied forward in time, others backward in time. The approach is based on a perturbation expansion in the strength of the coupling between forward and backward variables, and is well suited for situations in which initial and final conditions are imposed on different components of the system and the coupling between those components is weak. The form of the stochastic equations in our approach is determined by requiring that they generate the same statistics obtained in a forward description of the dynamics.

Numerical tests are carried out on a few simple two-degree-of-freedom systems. The merit and the difficulties of the approach are discussed and compared to more traditional strategies based on transition path sampling and simple shooting algorithms.
\end{abstract}

PACS numbers: 05.10.Gg,05.40.-a,05.10.Ln

\section{INTRODUCTION}

Stochastic systems are described by equations whose form depend on how the state of the system is measured, meaning, whether in an experiment, knowledge of the past state of the system or some different condition is assumed. The simplest example is provided by the Langevin dynamics

$$
\dot{E}+\Gamma E \mathrm{~d} t=\xi, \quad\langle\xi(t) \xi(0)\rangle=2 \mathcal{E} \delta(t),
$$

in which the sign of the relaxation coefficient $\Gamma$ depends on whether we are interested in determining the future evolution, or, as in backward induction [1], the previous history of the system. In the two cases, the solution of the differential equation can be expressed as an integral over past (future) values of the noise $\xi$, corresponding to causal (anti-causal) response of the system to external forcing [2]. In mathematical language, one says that the stochastic process is adapted to the forward (backward) filtration induced by the Wiener process associated with $\xi[3]$. The situation differs from the one in deterministic (Newtonian) mechanics, in which the form of the equations does not depend on the experiment one wishes to carry out on the system.

A forward description allows us in a natural way to take into account initial conditions. In the same way, a backward description allows us more easily to take into account final conditions. It is possible, however, to imagine more general-nonadapted-descriptions of the stochastic dynamics. In fact, there are infinite such descriptions; in the case of Eq. (11), for instance, the same dynamics could be generated by any equations in the form, in frequency space, $G_{\omega} E_{\omega}=\xi_{\omega},\left\langle\xi_{\omega} \xi_{\omega^{\prime}}\right\rangle=$ $4 \pi \mathcal{E} \delta\left(\omega+\omega^{\prime}\right)$, where $\left|G_{\omega}\right|^{2}=\Gamma^{2}+\omega^{2}$.

It has been suggested that nonadapted stochastic processes could be used to describe a stochastic dynamics conditioned at multiple times [4], and new forms of

\footnotetext{
* Email address for correspondence: olla@dsf.unica.it
}

stochastic calculus have been introduced to deal with this type of problems [5]. The simplest example of a conditioned stochastic system is the Brownian bridge, i.e. a Brownian motion in which the initial and final positions of the particle are supposedly given. The problem was initially studied by Schrödinger in the context of a possible interpretation of quantum mechanics as a statistical theory with boundary conditions in the past and the future [6]. The concept was later extended to more general systems, typically described by stochastic differential equations (SDEs), with applications ranging from mathematical finance [7], to ecology [8], to optimization theory [9]. Such extensions of the concept of a Brownian bridge are sometimes referred as a stochastic bridge [10].

The mathematical theory for the solution of conditioned SDE's is well established [11]. In practice, however, such problems require a sampling procedure, which can be numerically demanding if the number of variables is large, or if the final condition is a low probability state for the system. It is still possible, through a Doob transform [12, 13], to write a forward SDE with a modified drift that steers trajectories to the imposed final conditions, but the new drift depends on the same probability of the state of the system conditioned to its final state that one aims to determine. To overcome such difficulties, various methods have been devised, either based on some form of importance sampling 14 16], or on the adoption of transition path sampling algorithms [17, 18]

In this paper, we focus on a particular version of an "incomplete" stochastic bridge, in which initial and final conditions are imposed separately on different subsets of the variable describing the system. In a large deviation context [19, 20], e.g. one may be interested in studying the development of a large fluctuation in a particular region of a system, conditioned to the occurrence of some other event, say, the decay of another large fluctuation elsewhere in the system. Situations of physical interest include protein folding [21], chemical reactions [22, 23], as well as extreme events in the atmosphere [24]. More specifically, one may wish, e.g. to have an indicator of the occurrence of a future rare event in a component of 
a system (say, a heat wave in a particular region of the planet), from the modification of the dynamics in another portion of the system (the atmospheric circulation in another part of the globe). The matter has some intriguing aspects, as the interaction of portions of a system where large fluctuations are developing or decaying can be seen as the interaction between portions of the system with opposite (local) arrows of time: positive where the fluctuation is decaying, negative where the fluctuation is developing. Similar issues were studied in a cosmological context in 25].

Incomplete stochastic bridges provide an example of systems for which a nonadapted description can cure most difficulties generated by low probability final conditions. What we want to explore is the possibility of a description, which, in the limit of vanishing interaction between components of the system with opposite conditioning, becomes forward for the part of the system conditioned in the past and backward for the part conditioned in the future. For finite interaction, the description of the two components will not be purely forward or backward, and the system response to external stimuli will not have well-defined causality properties; what is lost because of the lack of a physical causal framework, however, is gained witha low probability final conditions being treated perturbatively around a backward description of the dynamics, which by construction does not require sampling. We shall speak in this case, of a mixed backward-forward description of the dynamics (concisely, a mixed description), to be opposed to the standard forward description afforded by Eq. (1), and to its time-reversed backward version.

We are going to limit our analysis to reversible systems. The simplest example is that of two bodies exchanging heat with one another and with a thermostat, a process that in recent years has attracted the attention of researchers interested in fluctuation theorems [26, 27], and calorimetric experiments in mesoscopic systems [28, 29].

It is interesting to note that in a mixed description, the two bodies will see the heat flowing, relative to their own time, from one to the other with an identical sign, i.e., simultaneously in or out from both. Such a condition could not be accommodated by a change of sign of a relaxation coefficient, as in the shift from Eq. (1) to its time-reversed version; a non-trivial redefinition of the concept of heat exchange is required.

The paper is organized as follows. In Sec. II, the mixed backward-forward approach is introduced by considering an infinite time horizon. Section III examines the problem of boundary conditions. In Sec. IV, the approach is applied to the case of linear dynamics. In Sec. V, the possible application of the technique to Monte Carlo evaluation of statistical quantities is discussed, and some applications and tests are presented. Section VI is devoted to the conclusions. Technical details are discussed in Appendixes $\mathrm{A}-\mathrm{C}$.

\section{MIXED BACKWARD-FORWARD APPROACH}

We consider the simplest possible example of a system with two degrees of freedom $E_{1,2}$ weakly coupled through correlation in the noise and an interaction component in the drift. The dynamics is described in the forward picture by the Itô SDE

$$
\dot{E}_{j}+F_{j}(\mathbf{E})=\xi_{j}, \quad\left\langle\xi_{j}(t) \xi_{k}(0)\right\rangle=2 \mathcal{E} \Xi_{j k} \delta(t),
$$

where

$$
F_{j}(\mathbf{E})=F_{j}^{(0)}\left(E_{j}\right)+g F_{j}^{(1)}(\mathbf{E}),
$$

and

$$
\Xi_{11}=\Xi_{22}=1, \quad \Xi_{12}=\Xi_{21}=-g
$$

We assume a reversible dynamics, which means that $X_{j k} F_{k} \equiv\left(\Xi^{-1}\right)_{j k} F_{k}$ must be a gradient [30]. This implies

$$
\left(X_{1 j} \partial_{E_{2}}-X_{2 j} \partial_{E_{1}}\right) F_{j}^{(1)}=0 .
$$

From Eq. (4) we find

$$
X_{11}=X_{22}=\frac{1+g}{1+2 g}, \quad X_{12}=X_{21}=\frac{g}{1+2 g},
$$

and by combining with Eq. (5),

$$
\begin{aligned}
F_{1}^{(1)}(\mathbf{E}) & =-F_{2}^{(1)}(\mathbf{E}) \\
& =F_{1}^{(0)}\left(E_{1}\right)-F_{2}^{(0)}\left(E_{2}\right)+H\left(E_{1}-E_{2}\right) .
\end{aligned}
$$

We seek a mixed backward-forward representation of the dynamics in Eqs. (2,7) as a perturbation expansion in $g$, which we will content ourselves to carry out up to first order. The stochastic equations in the mixed picture can be written in the form

$$
\begin{aligned}
& \dot{E}_{j}+M_{j}=\xi_{j}, \quad\left\langle\xi_{j}(t) \xi_{k}(0)\right\rangle=2 \mathcal{E} \Xi_{j k} \delta(t), \\
& M_{j}=M_{j}^{(0)}+g M_{j}^{(1)}+\ldots,
\end{aligned}
$$

where to lowest order we impose

$$
M_{1}^{(0)}=F_{1}^{(0)}\left(E_{1}\right), \quad M_{2}^{(0)}=-F_{2}^{(0)}\left(E_{2}\right) .
$$

Note that we have assumed identical statistics for the noise in the two pictures, a condition which is interpreted as a requirement of invariance for changes of stochastic description at the scale of the noise correlation time.

To determine the interaction terms $M_{j}^{(1)}$, we require identity of the statistics in the two representations, $\rho_{F}[\mathbf{E}]=\rho_{M}[\mathbf{E}]$, where $\rho_{F}[\mathbf{E}]$ and $\rho_{M}[\mathbf{E}]$ are the functional probability density functions (PDF) for the trajectories in the two pictures 31 33]. Adoption of the Itô prescription in the forward description guarantees that we do not have to care about Jacobian factors in the PDF:

$$
\begin{aligned}
\rho_{F}[\mathbf{E}] & =\mathcal{N} \exp \left[-\frac{1}{2 \mathcal{E}} \int \mathrm{d} t \mathcal{L}_{F}\right] \\
\mathcal{L}_{F} & =\frac{1}{2} X_{j k}\left(\dot{E}_{j}+F_{j}\right)\left(\dot{E}_{k}+F_{k}\right) .
\end{aligned}
$$


Since in this work we are not going to discuss how the system response to external forcing changes from one description to the other, we are not going to adopt a twofield representation for $\rho$, such as in the standard MartinSiggia-Rose approach [34].

To determine the PDF in the mixed picture, we need to select a prescription on the stochastic differentials. We interpret Eq. (8) at the discrete scale as

$$
\begin{gathered}
\Delta_{+} E_{1}(n)+M_{1}(n) \Delta t=\Delta_{+} W_{1}(n), \\
\Delta_{-} E_{2}(n)-M_{2}(n) \Delta t=\Delta_{-} W_{2}(n),
\end{gathered}
$$

where $\Delta_{ \pm}$indicate forward and backward time increments

$$
\Delta_{ \pm} f(n)=f(n \pm 1)-f(n),
$$

and $\Delta_{ \pm} W_{1,2}$ are Wiener increments, on which we impose cross correlations at lagged times:

$$
\begin{aligned}
& \left\langle\left(\Delta_{+} W_{1}\right)^{2}\right\rangle=\mathcal{E} \Xi_{11} \Delta t, \quad\left\langle\left(\Delta_{-} W_{2}\right)^{2}\right\rangle=\mathcal{E} \Xi_{22} \Delta t, \\
& \left\langle\Delta_{+} W_{1}(n) \Delta_{-} W_{2}(n+1)\right\rangle=-\mathcal{E} \Xi_{12} \Delta t .
\end{aligned}
$$

The adopted prescription reduces, in the decoupled limit $g \rightarrow 0$, to an Itô prescription for $E_{1}$ and a final point prescription for $E_{2}$, the last one equivalent to an Itô prescription in the backward time $t_{B}=T-t$ ( $T$ arbitrary). This tells us that for $g \rightarrow 0$, the PDF $\rho_{M}[\mathbf{E}]$ does not contain a Jacobian factor. For finite $g$, however, $\Delta E_{1}(n)$ and $\Delta E_{2}(n)$ receive contributions from terms $E_{2}(n+1)$ and $E_{1}(n-1)$, anticipating with respect to $t$ in one case, with respect to $t_{B}$ in the other. A nontrivial Jacobian contribution $J[\mathbf{E}]=\left|\operatorname{det}\left[\partial \Delta_{ \pm} W_{j}(n) / \partial E_{k}(m)\right]\right|$ is therefore expected. We derive the form of this contribution in Appendix A. The result of the calculation is given in Eq. (A8), and can be expressed in continuous time as follows:

$$
\begin{aligned}
J & =\exp \left[-\frac{g}{2 \mathcal{E}} \int \mathrm{d} t \mathcal{L}_{J}\right], \\
\mathcal{L}_{J}(t) & =-2 \mathcal{E}\left[\int_{t}^{+\infty} \mathrm{d} t^{\prime} \frac{\delta M_{1}^{(1)}\left(t^{\prime}\right)}{\delta E_{1}(t)}\right. \\
& \left.-\int_{-\infty}^{t} \mathrm{~d} t^{\prime} \frac{\delta M_{2}^{(1)}\left(t^{\prime}\right)}{\delta E_{2}(t)}\right]+O(g) .
\end{aligned}
$$

The procedure to obtain $\rho_{M}$ from the PDF for the noise history is identically to that in the forward case, and the result is

$$
\begin{aligned}
\rho_{M}[\mathbf{E}] & =\mathcal{N} \exp \left[-\frac{1}{2 \mathcal{E}} \int \mathrm{d} t\left(\mathcal{L}_{M}+g \mathcal{L}_{J}\right)\right], \\
\mathcal{L}_{M} & =\frac{1}{2} X_{j k}\left(\dot{E}_{j}+M_{j}\right)\left(\dot{E}_{k}+M_{k}\right),
\end{aligned}
$$

where $\dot{E}_{1}+M_{i} \equiv \Delta_{+} E_{1}(n) / \Delta t+M_{1}(n)$ and $\dot{E}_{2}+M_{2} \equiv$ $\Delta_{+} E_{2}(n) / \Delta t+M_{2}(n+1)$.

Identity of the statistics in the forward and mixed pictures is established through

$$
\mathcal{L}_{F}=\mathcal{L}_{M}+g \mathcal{L}_{J} .
$$

Substitution of Eqs. (10) and (15,16) into Eq. (17) yields an equation involving time derivatives $\dot{E}_{j}$. To deal with such terms, we recall the stochastic integration by parts formula

$$
X_{i j} \frac{\mathrm{d} V_{j} E_{j}}{\mathrm{~d} t}=X_{j k}\left[\dot{E}_{k} V_{k}+E_{k} \dot{V}_{j}\right]+\mathcal{E} \partial_{E_{j}} V_{j},
$$

which is a consequence of Itô's lemma [3].

Let us consider first the $O\left(g^{0}\right)$ part of Eq. (17). To use Eq. (18), we must convert all terms in Eq. (17) to a common Itô prescription. We have from Eqs. (6), (9), (10) and (16):

$$
\begin{aligned}
\mathcal{L}_{M}^{(0)}-\mathcal{L}_{F}^{(0)} & =\left[F_{2}^{(0)}(n)+F_{2}^{(0)}(n+1)\right] \frac{\Delta_{-} E_{2}(n+1)}{\Delta t} \\
& =-2 \dot{E}_{2} F_{2}^{(0)}\left(E_{2}\right)+\mathcal{E} \partial_{E_{2}} F_{2}^{(0)}\left(E_{2}\right)
\end{aligned}
$$

where the second line in the equation is understood in the Itô sense, and the last term is a Zakai-Wong correction [3]. Exploiting Eq. (18), with $\mathbf{V}=\left(0, F_{2}^{(0)}\right)$ and $X_{22}^{(0)}=1$, yields the expression

$$
\mathcal{L}_{M}^{(0)}-\mathcal{L}_{F}^{(0)}=-\frac{\mathrm{d} F_{2}^{(0)} E_{2}}{\mathrm{~d} t},
$$

which can then be eliminated from Eq. (17), at the price of a redefinition of the probability of states at $t= \pm \infty$.

We repeat the operations leading to Eq. (19), with the $O(g)$ remnant of Eq. (17). After substitution into Eq. (17), of Eqs. (6+7), (10) and (16), and after some straightforward algebra, we reach the result

$$
\begin{aligned}
& {\left[E_{1}(n) \frac{\mathrm{d}}{\mathrm{d} t}-F_{1}^{(0)}\right] \hat{M}_{1}(n) } \\
+ & {\left[E_{2}(n+1) \frac{\mathrm{d}}{\mathrm{d} t}+F_{2}^{(0)}\right] \hat{M}_{2}(n) } \\
= & 2\left(F_{1}^{(0)}+H\right) F_{2}^{(0)}+\mathcal{L}_{P}+\mathcal{L}_{I}+\mathcal{L}_{J},
\end{aligned}
$$

where the discrete scale shorthand $\mathrm{d} f(n) / \mathrm{d} t \equiv \dot{f}(n)=$ $\Delta_{+} f(n) / \Delta t$ continues to be used, we have defined

$$
\begin{aligned}
\hat{M}_{1}(n) & =M_{1}^{(1)}(n) \\
& -F_{1}^{(0)}(n)-F_{2}^{(0)}(n+1)-H(n), \\
\hat{M}_{2}(n+1) & =M_{1}^{(1)}(n+1) \\
& +F_{1}^{(0)}(n)+F_{2}^{(0)}(n+1)+H(n),
\end{aligned}
$$

together with

$$
\begin{aligned}
\mathcal{L}_{P}(n) & =-2 \dot{E}_{1}(n)\left[F_{2}^{(0)}(n)+F_{2}^{(0)}(n+1)\right] \\
& +2 \dot{E}_{1}(n)\left[F_{2}^{(0)}(n)-F_{2}^{(0)}(n+1)\right]
\end{aligned}
$$

and

$$
\mathcal{L}_{I}(n)=-\mathcal{E} \partial_{E_{j}}(n) \hat{M}_{j}(n),
$$

and we recall $H=H\left(E_{1}-E_{2}\right)$ is the part of the interaction explicitly dependent on the difference $E_{1}-E_{2}$ [see Eq. (7)]. The term $\mathcal{L}_{P}$ in Eq. (20) arises from the discrepancies in the prescriptions in Eqs. (11) and (12); the term $\mathcal{L}_{I}$ in the same equation is the Itô correction arising from application of Eq. (18) to the operation of shifting time derivatives in the terms $\dot{E}_{1,2} \hat{M}_{1,2}$ in $\mathcal{L}_{F}^{(1)}-\mathcal{L}_{M}^{(1)}$. We 
verify in Appendix $\mathrm{B}$ that $\mathcal{L}_{P}, \mathcal{L}_{I}$ and $\mathcal{L}_{J}$ can all be disregarded. Back to continuous time, Eq. (20) becomes then equivalent to the system of differential equations

$$
\begin{aligned}
& \left(\frac{\mathrm{d}}{\mathrm{d} t}-\frac{F_{1}^{(0)}}{E_{1}}\right) \hat{M}_{1}=\frac{F_{1}^{(0)} F_{2}^{(0)}}{E_{1}}+E_{2} Q, \\
& \left(\frac{\mathrm{d}}{\mathrm{d} t}+\frac{F_{2}^{(0)}}{E_{2}}\right) \hat{M}_{2}=\frac{F_{2}^{(0)}}{E_{2}}\left(F_{1}^{(0)}+2 H\right)-E_{1} Q,
\end{aligned}
$$

where $Q=Q(\mathbf{E}, t)$ is arbitrary. We can solve Eqs. (26) and (26) explicitly. By introducing

$$
\begin{aligned}
R_{1,2}(t) & =F_{1,2}^{(0)}\left(E_{1,2}(t)\right) / E_{1,2}(t), \\
S_{1}(t) & =R_{1}(t) F_{2}^{(0)}\left(E_{2}(t)\right)+E_{2}(t) Q(t), \\
S_{2}(t) & =R_{2}(t)\left[F_{1}^{(0)}\left(E_{1}(t)\right)\right. \\
& \left.+2 H\left(E_{1}(t)-E_{2}(t)\right)\right]-E_{1}(t) Q(t),
\end{aligned}
$$

we can write

$$
\begin{aligned}
& \hat{M}_{1}(t)=-\int_{t}^{+\infty} \mathrm{d} \tau S_{1}(t) \exp \left[-\int_{t}^{\tau} \mathrm{d} \tau^{\prime} R_{1}\left(\tau^{\prime}\right)\right], \\
& \hat{M}_{2}(t)=\int_{-\infty}^{t} \mathrm{~d} \tau S_{2}(t) \exp \left[-\int_{\tau}^{t} \mathrm{~d} \tau^{\prime} R_{2}\left(\tau^{\prime}\right)\right]
\end{aligned}
$$

We see that different choices of the arbitrary function $Q(\mathbf{E}, t)$ allow us to shift the weight of the nonlocal contribution to the dynamics between $\hat{M}_{1}$ and $\hat{M}_{2}$.

We summarize the main results of the section. The stochastic system is described in the mixed picture by a system of stochastic equations [Eqs. (8) and (9)], involving new variables $\hat{M}_{j}$ [Eqs. (21]22) and (25]|26)] that are anticipating relative to the proper time of the respective component: the forward time $t$ for $j=1$, the backward time $t_{B}=T-t$ for $j=2$. The new description thus becomes nonlocal in time, a situation which becomes manifest if the new variables are expressed as functionals of the old ones through Eqs. (30,31), thus turning Eq. (8) into a system of integrodifferential stochastic equations.

The upshot is that a naive perturbative approach based on an iterative solution of purely forward and backward equations in separate portions of the system, using at each iteration the values of the variables outside that portion, obtained in the previous iteration would not work; modified equations must be used, whose form at $O(g)$ is given in Eqs. (8), (9), (21) 222) and (25) 26).

\section{TREATMENT OF BOUNDARY CONDITIONS}

We have derived the stochastic equations in the mixed picture, Eqs. (8), (9), (21,22) and (25]|26), in an infinite time domain. For the same reason a forward SDE in the form of Eq. (1) cannot directly be utilized to take into account final conditions, the equations derived in the previous section cannot be used, in their current form, for the treatment of boundary condition problems.
We consider the following boundary conditions

$$
\mathcal{B} \equiv\left\{\mathcal{B}_{1}, \mathcal{B}_{2}\right\}=\left\{E_{1}\left(t_{i}\right)=E_{1}^{i}, E_{2}\left(t_{f}\right)=E_{2}^{f}\right\},
$$

which provide us with the simplest example of incomplete stochastic bridge.

A natural extension of the approach in Sec. [I] would be to solve perturbatively Eq. (8),, $\mathbf{E}=\mathbf{E}^{(0)}+g \mathbf{E}^{(1)}+\ldots$, and to enforce Eq. (32) order by order in the expansion. Unfortunately, while it is straightforward to impose Eq. (32) on $\mathbf{E}^{(0)}$, we have no control on $\mathbf{E}^{(1)}$; in fact, $E_{1}^{(1)}\left(t_{i}\right)$ and $E_{2}^{(1)}\left(t_{f}\right)$ are determined by the behavior of $\mathbf{E}$ outside $\left[t_{i}, t_{f}\right]$, and their value is in general non-zero. The evaluation of statistical quantities must therefore include correction terms accounting for the fact that the boundary conditions in Eq. (32) can only be enforced to $O\left(g^{0}\right)$.

The algebra is somewhat lighter if instead of expanding in $g$, we decompose $\mathbf{E}$ in contributions from the noise and from the $O(g)$ part of the drift:

$$
\mathbf{E} \simeq \overline{\mathbf{E}}+g \tilde{\mathbf{E}}+O\left(g^{2}\right),
$$

where $\overline{\mathbf{E}}$ and $\tilde{\mathbf{E}}$ obey

$$
\dot{\bar{E}}_{j}+M_{j}^{(0)}=\xi_{j}
$$

and

$$
\dot{\tilde{E}}_{j}+\tilde{E}_{k} \partial_{\bar{E}_{k}} M_{j}^{(0)}+M_{j}^{(1)}=0 .
$$

Note that since $\bar{E}_{1}$ and $\bar{E}_{2}$ only depend on the past (future) history of the noise, the following condition of statistical independence is going to hold:

$$
\rho_{M}\left(\bar{E}_{1}(t) \bar{E}_{2}\left(t^{\prime}\right)\right)=\rho_{M}\left(\bar{E}_{1}\right) \rho_{M}\left(\bar{E}_{2}\right), \quad t^{\prime}>t .
$$

The condition does not hold in the forward picture, therefore $\rho_{M}[\overline{\mathbf{E}}] \neq \rho_{F}[\overline{\mathbf{E}}]$. Since all the following calculations are carried out in the mixed picture, however, for lighter notation, we shall drop subscript $M$ on all averages and PDF's involving $\overline{\mathbf{E}}$.

The statistics of the conditioned problem is contained in the generating functional

$$
\begin{aligned}
Z[\mathbf{J}] & =\left\langle\exp \left[\mathrm{i} \int \mathrm{d} t \mathbf{J}(t) \cdot \mathbf{E}(t)\right] \mid \mathcal{B}\right\rangle \\
& =\mathcal{Q}\left\langle\delta_{\mathcal{B}} \exp \left[\mathrm{i} \int \mathrm{d} t \mathbf{J}(t) \cdot \mathbf{E}(t)\right]\right\rangle,
\end{aligned}
$$

where

$$
\mathcal{Q}^{-1}=\left\langle\delta_{\mathcal{B}}\right\rangle \equiv \rho(\mathcal{B}),
$$

and $\delta_{\mathcal{B}}$ is the Dirac delta enforcing the boundary condition $\mathcal{B}$.

Let us indicate

$$
\overline{\mathcal{B}} \equiv\left\{\overline{\mathcal{B}}_{1}, \overline{\mathcal{B}}_{2}\right\}=\left\{\bar{E}_{1}\left(t_{i}\right)=E_{1}^{i}, \bar{E}_{2}\left(t_{f}\right)=E_{2}^{f}\right\},
$$

and decompose $Z$ as in Eq. (33),

$$
Z[\mathbf{J}]=\bar{Z}[\mathbf{J}]+g \tilde{Z}[\mathbf{J}]+O\left(g^{2}\right),
$$


where

$$
\begin{aligned}
\bar{Z}[\mathbf{J}] & =\left\langle\exp \left[\mathrm{i} \int \mathrm{d} t \mathbf{J}(t) \cdot \mathbf{E}(t)\right] \mid \overline{\mathcal{B}}\right\rangle \\
& =\overline{\mathcal{Q}}\left\langle\delta_{\overline{\mathcal{B}}} \exp \left[\mathrm{i} \int \mathrm{d} t \mathbf{J}(t) \cdot \mathbf{E}(t)\right]\right\rangle, \\
\overline{\mathcal{Q}}^{-1} & =\left\langle\delta_{\overline{\mathcal{B}}}\right\rangle \equiv \rho(\overline{\mathcal{B}}),
\end{aligned}
$$

and

$$
\begin{aligned}
g \tilde{Z}[\mathbf{J}] & =\left(\frac{\mathcal{Q}}{\overline{\mathcal{Q}}}-1\right)\left\langle\exp \left[\mathrm{i} \int \mathrm{d} t \mathbf{J}(t) \cdot \overline{\mathbf{E}}(t)\right] \mid \overline{\mathcal{B}}\right\rangle \\
& +\overline{\mathcal{Q}}\left\langle\left(\delta_{\mathcal{B}}-\delta_{\overline{\mathcal{B}}}\right) \exp \left[\mathrm{i} \int \mathrm{d} t \mathbf{J}(t) \cdot \overline{\mathbf{E}}(t)\right]\right\rangle .
\end{aligned}
$$

Thus, $\bar{Z}[\mathbf{J}]$ contains the statistics that would be obtained if the boundary conditions were enforced on $\overline{\mathbf{E}}$ instead of $\mathbf{E}$, and $\tilde{Z}[\mathbf{J}]$ contains the correction.

The correction term $\tilde{Z}[\mathbf{J}]$ is evaluated in Appendix C] Let us introduce the notation $\left\langle\tilde{\mathbf{E}} \exp \left(\mathrm{i} \int \mathbf{J} \cdot \mathbf{E}\right) \mid \overline{\mathcal{B}}\right\rangle^{c} \equiv$ $\left\langle\tilde{\mathbf{E}} \exp \left(\mathrm{i} \int \mathbf{J} \cdot \mathbf{E}\right) \mid \overline{\mathcal{B}}\right\rangle-\langle\tilde{\mathbf{E}} \mid \overline{\mathcal{B}}\rangle\left\langle\exp \left(\mathrm{i} \int \mathbf{J} \cdot \mathbf{E}\right) \mid \overline{\mathcal{B}}\right\rangle$, and indicate

$$
\begin{aligned}
& \nabla_{i f}=\left(\partial_{E_{1}^{i}}, \partial_{E_{2}^{f}}\right), \quad \tilde{\mathbf{E}}_{i f}=\left(\tilde{E}_{1}\left(t_{i}\right), \tilde{E}_{2}\left(t_{f}\right)\right), \\
& \mathbf{F}_{i f}^{(0)}=\left(F_{1}^{(0)}\left(E_{1}^{i}\right), F_{2}^{(0)}\left(E_{2}^{f}\right)\right) .
\end{aligned}
$$

A calculation detailed in Appendix C allows us then to evaluate the correction term $\tilde{Z}[\mathbf{J}]$ as follows

$$
\begin{aligned}
\tilde{Z}[\mathbf{J}] & =\left[\frac{\mathbf{F}_{i f}^{(0)}}{\mathcal{E}}-\nabla_{i f}\right] \cdot\left\langle\tilde{\mathbf{E}}_{i f} \exp \left[\mathrm{i} \int \mathrm{d} t \mathbf{J}(t) \cdot \overline{\mathbf{E}}(t)\right] \mid \overline{\mathcal{B}}\right\rangle^{c} \\
& -\left\langle\tilde{\mathbf{E}}_{i f} \mid \overline{\mathcal{B}}\right\rangle \cdot \nabla_{i f}\left\langle\exp \left[\mathrm{i} \int \mathrm{d} t \mathbf{J}(t) \cdot \overline{\mathbf{E}}(t)\right] \mid \overline{\mathcal{B}}\right\rangle \cdot
\end{aligned}
$$

Substituting Eqs. (41) and (45) into Eq. (40) produces the final expression

$$
\begin{aligned}
Z[\mathbf{J}] & =\left[1-g\left\langle\tilde{\mathbf{E}}_{i f} \mid \overline{\mathcal{B}}\right\rangle \cdot \nabla_{i f}\right]\left\langle\exp \left[\mathrm{i} \int \mathrm{d} t \mathbf{J}(t) \cdot \mathbf{E}(t)\right] \mid \overline{\mathcal{B}}\right\rangle \\
& +g\left[\frac{\mathbf{F}_{i f}^{(0)}}{\mathcal{E}}-\nabla_{i f}\right] \cdot\left\langle\tilde{\mathbf{E}}_{i f} \exp \left[\mathrm{i} \int \mathrm{d} t \mathbf{J}(t) \cdot \overline{\mathbf{E}}(t)\right] \mid \overline{\mathcal{B}}\right\rangle^{c} \\
& +O\left(g^{2}\right) .
\end{aligned}
$$

From Eq. (46) we can obtain expressions for the conditional averages $\left\langle\bar{E}_{1,2} \mid \overline{\mathcal{B}}\right\rangle$. We can exploit Eq. (36) to write $\left\langle\bar{E}_{1,2} \mid \overline{\mathcal{B}}\right\rangle=\left\langle\bar{E}_{1,2} \mid \overline{\mathcal{B}}_{1,2}\right\rangle$. We introduce the notation for the fluctuations

$$
\mathbf{e}(t)=\mathbf{E}(t)-\langle\mathbf{E}(t) \mid \overline{\mathcal{B}}\rangle,
$$

with similar definitions holding for $\overline{\mathbf{e}}$ and $\tilde{\mathbf{e}}_{i f}$, and find

$$
\begin{aligned}
\left\langle E_{1}(t) \mid \mathcal{B}\right\rangle & =\left[1-g\left\langle\tilde{E}_{1}\left(t_{i}\right) \mid \overline{\mathcal{B}}\right\rangle \partial_{E_{1}^{i}}\right]\left\langle\bar{E}_{1}(t) \mid \overline{\mathcal{B}}_{1}\right\rangle \\
& +g\left[\frac{\mathbf{F}_{i f}^{(0)}}{\mathcal{E}}-\nabla_{i f}\right] \cdot\left\langle\tilde{\mathbf{e}}_{i f} \bar{e}_{1}(t) \mid \overline{\mathcal{B}}\right\rangle \\
& +g\left\langle\tilde{E}_{1}(t) \mid \overline{\mathcal{B}}\right\rangle+O\left(g^{2}\right), \\
\left\langle E_{2}(t) \mid \mathcal{B}\right\rangle & =\left[1-g\left\langle\tilde{E}_{2}\left(t_{f}\right) \mid \overline{\mathcal{B}}\right\rangle \partial_{E_{2}^{f}}\right]\left\langle\bar{E}_{2}(t) \mid \overline{\mathcal{B}}_{2}\right\rangle \\
& +g\left[\frac{\mathbf{F}_{i f}^{(0)}}{\mathcal{E}}-\nabla_{i f}\right] \cdot\left\langle\tilde{\mathbf{e}}_{i f} \bar{e}_{2}(t) \mid \overline{\mathcal{B}}\right\rangle \\
& +g\left\langle\tilde{E}_{2}(t) \mid \overline{\mathcal{B}}\right\rangle+O\left(g^{2}\right) .
\end{aligned}
$$

The presence of terms $\propto \mathcal{E}^{-1}\left\langle\tilde{\mathbf{e}}_{i f} \bar{e}_{j}(t) \mid \overline{\mathcal{B}}\right\rangle$ in Eqs. (47) and (48) tells us that fluctuations remain important also in a large deviation regime $\mathcal{E} \rightarrow 0$. It is worth pointing out that these terms produce the dominant contribution to the error in the numerical evaluation of $\langle\mathbf{E} \mid \mathcal{B}\rangle$. Indeed, the error in sample averages $\langle\overline{\mathbf{E}} \mid \overline{\mathcal{B}}\rangle_{N}$ and $\langle\tilde{\mathbf{E}} \mid \overline{\mathcal{B}}\rangle_{N}$ scales with $(\mathcal{E} / N)^{1 / 2}$, where $N$ is the sample size, while the one in $\mathcal{E}^{-1}\left\langle\tilde{\mathbf{e}} \bar{e}_{j} \mid \overline{\mathcal{B}}\right\rangle_{N}$ scales with $N^{-1 / 2}$.

\section{APPLICATION TO A LINEAR SYSTEM}

Consider the following system of SDE's:

$$
\begin{aligned}
& \dot{E}_{1}+E_{1}+g\left(E_{1}-E_{2}\right)=\xi_{1}, \\
& \dot{E}_{2}+E_{2}+g\left(E_{2}-E_{1}\right)=\xi_{2},
\end{aligned}
$$

with noise statistics obeying Eqs. (2) and (44). The equations, for $g>0$, could describe in the forward picture a pair of identical bodies that exchange heat with a thermostat and (weakly) with one another.

The equations describing the system dynamics in the mixed picture are obtained from Eqs. (8), (9), (21-22) and (30) 31). We note that $H=0$. In Eqs. (25) and (26) we set $Q=0$ [the most natural choice given the symmetry of Eqs. (49, [50)] and get

$$
\begin{aligned}
& \dot{E}_{1}+E_{1}+g\left(\hat{M}_{1}+E_{1}+E_{2}\right)+\ldots=\xi_{1}, \\
& \dot{E}_{2}-E_{1}+g\left(\hat{M}_{2}-E_{1}-E_{2}\right)+\ldots=\xi_{2},
\end{aligned}
$$

where

$$
\begin{aligned}
& \hat{M}_{1}(t)=-\int_{t}^{+\infty} \mathrm{d} \tau E_{2}(\tau) \mathrm{e}^{t-\tau}, \\
& \hat{M}_{2}(t)=\int_{-\infty}^{t} \mathrm{~d} \tau E_{1}(\tau) \mathrm{e}^{\tau-t} .
\end{aligned}
$$

As expected, the heat transfer between the two bodies loses in the mixed picture its original character of a relaxation process.

It is possible to verify that the statistics of $\mathbf{E}$ in the two pictures coincide - as requested by the theory - by showing that the expression of the correlation functions $\left\langle E_{j}(t) E_{k}\left(t^{\prime}\right)\right\rangle$ obtained in the two cases is identical. The calculation is straightforward and is not carried out here. Instead, we direct our attention to the dynamics of the conditioned system, and verify that the forward and the mixed backward-forward approach lead to identical expressions for the average trajectories $\langle\mathbf{E}(t) \mid \mathcal{B}\rangle$.

Let us consider first the forward approach. Thanks to linearity, the average trajectories can be expressed as a superposition $\left\langle E_{j}(t) \mid \mathcal{B}\right\rangle=\left\langle E_{j}(t) \mid \mathcal{B}_{1}\right\rangle+\left\langle E_{j}(t) \mid \mathcal{B}_{2}\right\rangle$. From Eqs. (49) w0 we then get, for $t \in\left[t_{i}, t_{f}\right]$,

$$
\begin{aligned}
\left\langle E_{a}(t) \mid \mathcal{B}\right\rangle & =a_{+}\left(t-t_{i}\right) E_{a}^{i}+a_{-}\left(t_{f}-t\right) E_{b}^{f}, \\
\left\langle E_{b}(t) \mid \mathcal{B}\right\rangle & =a_{-}\left(t-t_{i}\right) E_{a}^{i}+a_{+}\left(t_{f}-t\right) E_{b}^{f},
\end{aligned}
$$


where $a_{ \pm}(t)=\left[\mathrm{e}^{-t} \pm \mathrm{e}^{-(1+2 g) t}\right] / 2$; expanding to first order in $g$ :

$$
\begin{aligned}
\left\langle E_{1}(t) \mid \mathcal{B}\right\rangle & =E_{1}^{i}\left[1-g\left(t-t_{i}\right)\right] \mathrm{e}^{t_{i}-t}+g E_{2}^{f}\left[\left(t_{f}-t\right) \mathrm{e}^{t-t_{f}}\right. \\
& \left.-\left(t_{f}-t_{i}\right) \mathrm{e}^{2 t_{i}-t-t_{f}}\right]+O\left(g^{2}\right), \\
\left\langle E_{2}(t) \mid \mathcal{B}\right\rangle & =E_{2}^{f}\left[1-g\left(t_{f}-t\right)\right] \mathrm{e}^{t-t_{f}}+g E_{1}^{i}\left[\left(t-t_{i}\right) \mathrm{e}^{t_{i}-t}\right. \\
& \left.-\left(t_{f}-t_{i}\right) \mathrm{e}^{t_{i}+t-2 t_{f}}\right]+O\left(g^{2}\right) .
\end{aligned}
$$

The same expressions could be obtained by a rather lengthy calculation from the Brownian bridge expression for the statistics $\rho(\mathbf{E}(t) \mid \mathcal{B})=$ $\rho\left(\mathcal{B}_{2} \mid \mathbf{E}(t)\right) \rho\left(\mathbf{E}(t) \mid \mathcal{B}_{1}\right) / \rho\left(\mathcal{B}_{2} \mid \mathcal{B}_{1}\right)[6]$.

To obtain the expression for $\langle\mathbf{E}(t) \mid \mathcal{B}\rangle$ in the mixed picture, we must evaluate the conditional averages $\langle\overline{\mathbf{E}} \mid \overline{\mathcal{B}}\rangle$, $\langle\tilde{\mathbf{E}} \mid \overline{\mathcal{B}}\rangle$ and the correlation $\left\langle\tilde{\mathbf{e}}_{i f} \bar{e}_{j} \mid \overline{\mathcal{B}}\right\rangle$.

By setting $F_{j}^{(0)}=E_{j}$ in Eqs. (34) and exploiting Eq. (36), we find

$$
\left\langle\bar{E}_{1}(t) \mid \overline{\mathcal{B}}\right\rangle=E_{1}^{i} \mathrm{e}^{t_{i}-t}, \quad\left\langle\bar{E}_{2}(t) \mid \overline{\mathcal{B}}\right\rangle=E_{2}^{f} \mathrm{e}^{t-t_{f}} .
$$

By setting $M_{1}^{(1)}=E_{1}+E_{2}+\hat{M}_{1}, M_{2}^{(1)}=-E_{1}-E_{2}+$ $\hat{M}_{2}$, with $\hat{M}_{j}$ given in Eqs. (53) (54), and substituting the result in (35), we find

$$
\begin{aligned}
\tilde{E}_{1}(t) & =-\int_{-\infty}^{t} \mathrm{~d} \tau \mathrm{e}^{\tau-t}\left[\bar{E}_{1}(\tau)+\frac{1}{2} \bar{E}_{2}(\tau)\right] \\
& +\frac{1}{2} \int_{t}^{+\infty} \mathrm{d} \tau \mathrm{e}^{t-\tau} \bar{E}_{2}(\tau)
\end{aligned}
$$

and

$$
\begin{aligned}
\tilde{E}_{2}(t) & =-\int_{t}^{+\infty} \mathrm{d} \tau \mathrm{e}^{t-\tau}\left[\bar{E}_{2}(\tau)+\frac{1}{2} \bar{E}_{1}(\tau)\right] \\
& +\frac{1}{2} \int_{-\infty}^{t} \mathrm{~d} \tau \mathrm{e}^{\tau-t} \bar{E}_{1}(\tau) .
\end{aligned}
$$

Substituting Eq. (59) into Eqs. 60 61) we then obtain

$$
\begin{aligned}
\left\langle\tilde{E}_{1}(t) \mid \overline{\mathcal{B}}\right\rangle & =-E_{1}^{i}\left(\frac{1}{2}+t-t_{i}\right) \mathrm{e}^{t_{i}-t} \\
& +\frac{E_{2}^{f}}{2}\left(t_{f}-t\right) \mathrm{e}^{t-t_{f}}, \\
\left\langle\tilde{E}_{2}(t) \mid \overline{\mathcal{B}}\right\rangle & =-E_{2}^{f}\left(\frac{1}{2}+t_{f}-t\right) \mathrm{e}^{t-t_{f}} \\
& +\frac{E_{1}^{i}}{2}\left(t-t_{i}\right) \mathrm{e}^{t_{i}-t} .
\end{aligned}
$$

The fluctuation contribution $\left\langle\tilde{\mathbf{e}}_{i f} \bar{e}_{j} \mid \overline{\mathcal{B}}\right\rangle$ is proportional, through Eqs. (60-61), to $\left\langle\bar{e}_{i}(t) \bar{e}_{j}\left(t^{\prime}\right) \mid \overline{\mathcal{B}}\right\rangle$. Thus, since $\left\langle\tilde{\mathbf{e}}_{i f} \bar{e}_{j} \mid \overline{\mathcal{B}}\right\rangle$ enters Eqs. (47]48) already at $O(g)$, we need to evaluate $\left\langle\bar{e}_{i}(t) \bar{e}_{j}\left(t^{\prime}\right) \mid \overline{\mathcal{B}}\right\rangle$ only to $O\left(g^{0}\right)$; it is thus irrelevant whether we work in the forward or in the backward picture. We immediately find $\left\langle\bar{e}_{1} \bar{e}_{1} \mid \overline{\mathcal{B}}\right\rangle^{(0)}=\left\langle\bar{e}_{1} \bar{e}_{1} \mid \overline{\mathcal{B}}_{1}\right\rangle^{(0)}$, $\left\langle\bar{e}_{2} \bar{e}_{2} \mid \overline{\mathcal{B}}\right\rangle^{(0)}=\left\langle\bar{e}_{2} \bar{e}_{2} \mid \overline{\mathcal{B}}_{2}\right\rangle^{(0)}$, and $\left\langle\bar{e}_{1} \bar{e}_{2} \mid \overline{\mathcal{B}}\right\rangle=O(g)$; the last term can therefore be disregarded. Since $e_{1}(t)$ and $e_{1}\left(t^{\prime}\right)$ are uncorrelated at opposite sides of $t_{i}$, and $e_{2}(t)$ and $e_{2}\left(t^{\prime}\right)$ are uncorrelated at opposite sides of $t_{f}$, the only contribution to $\langle\mathbf{E}(t) \mid \mathcal{B}\rangle$ comes from

$$
\left\langle\bar{e}_{1}(t) \bar{e}_{1}\left(t^{\prime}\right) \mid \overline{\mathcal{B}}\right\rangle^{(0)}=\frac{\mathcal{E}}{2}\left[\mathrm{e}^{-\left|t-t^{\prime}\right|}-\mathrm{e}^{-\left|t+t^{\prime}-2 t_{i}\right|}\right],
$$

with $t, t^{\prime}>t_{i}$ or $t, t^{\prime}<t_{i}$, and from

$$
\left\langle\bar{e}_{2}(t) \bar{e}_{2}\left(t^{\prime}\right) \mid \overline{\mathcal{B}}\right\rangle^{(0)}=\frac{\mathcal{E}}{2}\left[\mathrm{e}^{-\left|t-t^{\prime}\right|}-\mathrm{e}^{-\left|t+t^{\prime}-2 t_{f}\right|}\right],
$$

with $t, t^{\prime}>t_{f}$ or $t, t^{\prime}<t_{f}$. By exploiting Eqs. 60 61 we then get the result, for $t \in\left[t_{i}, t_{f}\right]$,

$$
\begin{aligned}
\left\langle\tilde{e}_{1}\left(t_{i}\right) \bar{e}_{2}(t) \mid \overline{\mathcal{B}}\right\rangle & =\frac{\mathcal{E}}{2}\left[\left(t-t_{i}\right) \mathrm{e}^{t_{i}-t}\right. \\
& \left.-\left(t_{f}-t_{i}\right) \mathrm{e}^{t_{i}+t-2 t_{f}}\right]+O(g), \\
\left\langle\tilde{e}_{2}\left(t_{f}\right) \bar{e}_{1}(t) \mid \overline{\mathcal{B}}\right\rangle & =\frac{\mathcal{E}}{2}\left[\left(t_{f}-t\right) \mathrm{e}^{t-t_{f}}\right. \\
& \left.-\left(t_{f}-t_{i}\right) \mathrm{e}^{2 t_{i}-t-t_{f}}\right]+O(g), \\
\left\langle\tilde{e}_{1}\left(t_{i}\right) \bar{e}_{1}(t) \mid \overline{\mathcal{B}}\right\rangle & \sim\left\langle\tilde{e}_{2}\left(t_{f}\right) \bar{e}_{2}(t) \mid \overline{\mathcal{B}}\right\rangle=O(g) .
\end{aligned}
$$

We can now substitute Eqs. (59), 62 63) and (66-67) into Eqs. (47,48), and verify with little algebra that the same expression for $\langle\mathbf{E} \mid \mathcal{B}\rangle$ in Eqs. (57, 58) is reproduced.

\section{NUMERICAL TESTS}

The numerical solution of the stochastic equations in the mixed picture has important peculiarities. Firstly, since different sets of variables are integrated into opposite time directions, the evaluation of the interaction terms require that the histories of the variables $\bar{E}_{j}$ be already calculated and stored in memory. Secondly, because of the intrinsic time non-local nature of the mixed picture, and the fact that boundary conditions in an incomplete stochastic bridge are imposed only on one part of the variables, the stochastic equations for a problem conditioned at $t_{i}$ and $t_{f}, t_{i}<t_{f}$, must be solved in a wider domain $\left[T_{i}, T_{f}\right] \supset\left[t_{i}, t_{f}\right]$.

The determination of the trajectories, to be used for the Monte Carlo evaluation of statistical quantities in Eq. (46), therefore, is going to proceed through the following sequence of steps:

- Generate a noise history $\boldsymbol{\xi}$ in $\left[T_{i}, T_{f}\right]$, and store it in memory.

- Obtain from $\boldsymbol{\xi}_{i}$ and store in memory the history of $\overline{\mathbf{E}}$ in the interval $\left[T_{i}, T_{f}\right]$ (at this point the history of $\boldsymbol{\xi}$ is not needed anymore). Since the whole history of $\overline{\mathbf{E}}$ in the whole interval $\left[t_{i}, t_{f}\right]$ is required, forward variables are going to become backward variables in $\left[T_{i}, t_{i}\right]$ and backward variables become forward variables in $\left[t_{f}, T_{f}\right]$. For the problem considered in Sec. III] this means that $\bar{E}_{1}$ and $\bar{E}_{2}$ are going to obey equations $\dot{\bar{E}}_{1}-F_{1}^{(0)}=\xi_{1}$ and $\dot{\bar{E}}_{2}+F_{2}^{(0)}=\xi_{2}$ in domains $\left[T_{i}, t_{i}\right]$ and $\left[t_{f}, T_{f}\right]$, respectively 


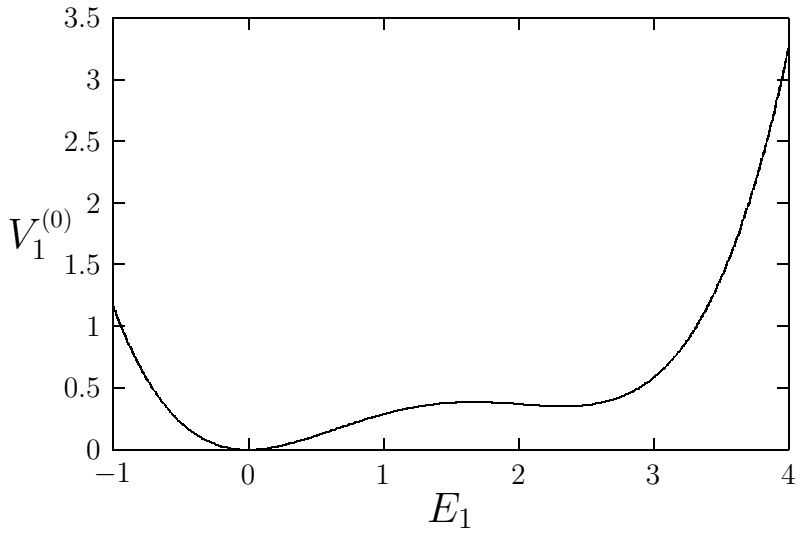

FIG. 1. Profile of the potential $V_{1}^{(0)}$ for system $c$.

- Again in $\left[T_{i}, T_{f}\right]$, obtain $\hat{\mathbf{M}}$ from $\overline{\mathbf{E}}$. For the system considered in Sec. III this is accomplished by integrating Eq. (25) backward and Eq. (26) forward in time. The domain $\left[T_{i}, T_{f}\right]$ must therefore be large enough to guarantee that the effect of the boundary conditions at $T_{i, f}$ on the profile of $\hat{\mathbf{M}}$ in $\left[t_{i}, t_{f}\right]$ be negligible. Since each $\tilde{E}_{j}$ is is obtained by integrating in the time direction opposite to that of the corresponding $\hat{M}_{j}$, also $\hat{\mathbf{M}}$ must be stored in memory.

- From $\overline{\mathbf{E}}$ and $\hat{\mathbf{M}}$, finally obtain also $\tilde{\mathbf{E}}$; this requires integrating the equations for the forward variables from $T_{i}$ to $t_{f}$ and those for the backward variables from $T_{f}$ to $t_{i}$. Once more, the domain $\left[T_{i}, T_{f}\right]$ must be chosen large enough for the boundary conditions on $\tilde{\mathbf{E}}$ at $T_{i, f}$ not to affect the behavior of the variables in the domain of interest $\left[t_{i}, t_{f}\right]$.

We continue to focus our attention on simple twodegree-of-freedom systems, and verify that the forward and the mixed picture generates identical statistics. We carry out our tests on the deviation of the average trajectories from the decoupled case:

$$
\langle\mathbf{E}(t) \mid \mathcal{B}\rangle^{\prime}=\langle\mathbf{E}(t) \mid \mathcal{B}\rangle-\langle\mathbf{E}(t) \mid \mathcal{B}\rangle^{(0)} .
$$

We consider three examples of stochastic system; the following profiles for the unperturbed drift are adopted:

$$
\begin{aligned}
& F_{1}^{(0)}=E_{1}, \quad \text { system } a, \\
& F_{1}^{(0)}=\frac{1}{6}\left(E_{1}^{3}+E_{1}\right), \quad \text { system } b, \\
& F_{1}^{(0)}=\frac{1}{3}\left(E_{1}^{3}-4 E_{1}^{2}+\frac{35}{9} E_{1}\right), \quad \text { system } c,
\end{aligned}
$$

with $F_{2}^{(0)}=E_{2}$ in the three cases. The statistics in Eq. (2) is utilized for the noise and the condition $H=0$ is imposed in the interaction term. The values of the remaining parameters are listed in Table I.

The potential $V_{1}^{(0)}, F_{1}^{(0)}=V_{1}^{(0) \prime}$, has for systems $a$ and $b$ the form a single quadratic (quartic) well; for system $c$,
TABLE I. Simulation parameters.

\begin{tabular}{ll}
\hline \hline$t_{i}=0$, & $t_{f}=3,($ system $a)$ \\
$T_{i}=-17$, & $T_{f}=20$, \\
$E_{1}^{i}=4$ & $E_{2}^{f}=1,($ system $a)$ \\
$\mathcal{E}=0.1$, & $g=0.1$, \\
$N=10^{5}$ (sample size). & \\
\hline \hline
\end{tabular}

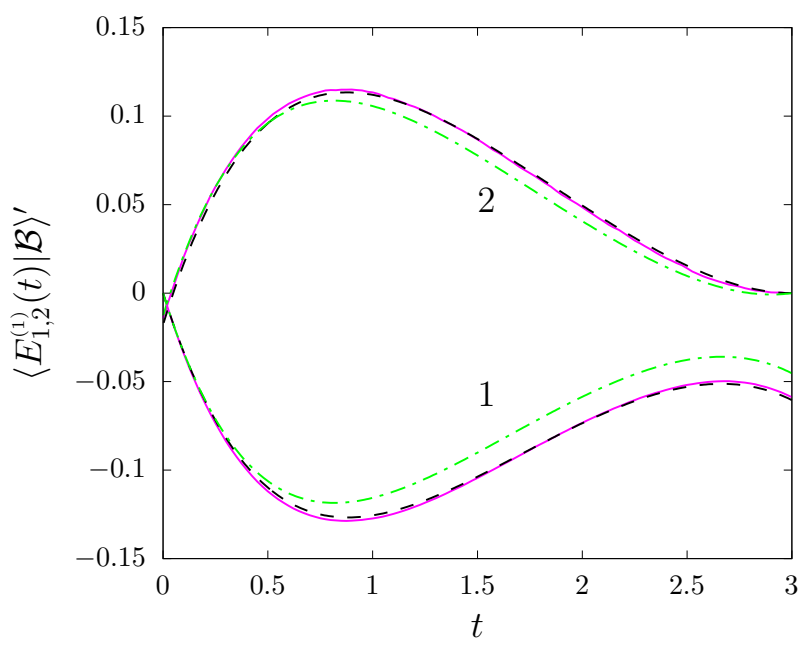

FIG. 2. Mean trajectory deviation from the decoupled limit in the case of system $a$. Result of the numerical integration continuous line (magenta online); exact profiles from Eqs. (55) d56) dash-dotted line (green online); analytical $O(g)$ profiles from Eqs. (57.58) black dashed line.

it is a rather shallow double potential well, with profile shown in Fig. 1.

In the case of system $a$, analytical expressions for the mean profiles of a generic incomplete stochastic bridge are available [Eqs. [57-58)]. As can be checked in Fig. 2. the result of numerical simulations basically overlap with the analytical expressions for the $O(g)$ profiles in Eq. (57, 58).

In the case of systems $b$ and $c$, we consider an initial condition problem, with $\mathcal{B}_{1}=\left\{E_{1}\left(t_{i}\right)=E_{1}^{i}\right\}$, and $E_{2}\left(t_{i}\right)$ distributed according to $\rho\left(E_{2}\left(t_{i}\right) \mid \mathcal{B}_{1}\right)$. This allows direct comparison of numerical simulations in the forward and mixed picture, without having to resort, in the forward case, to sampling.

In order to compare the result of simulations in the two pictures, the time separation $t_{f}-t_{i}$ must be sufficiently large to be able to approximate $\rho\left(E_{2}\left(t_{i}\right) \mid \mathcal{B}\right) \simeq$ $\rho\left(E_{2}\left(t_{i}\right) \mid \mathcal{B}_{1}\right)$. In alternative, $E_{2}\left(t_{f}\right)$ must be extracted from the PDF $\rho\left(E_{2}\left(t_{f}\right) \mid \mathcal{B}\right)$, which, to the order in $g$ considered, can be approximated with $\rho^{(0)}\left(E_{2}\left(t_{f}\right)\right) \sim$ $\exp \left[-\left(E_{2}\left(t_{i}\right)\right)^{2} /(2 \mathcal{E})\right]$ anyway. We note that for the dynamics considered the relation $\rho\left(E_{2}\left(t_{i}\right)\right)=\rho^{(0)}\left(E_{2}\left(t_{i}\right)\right) \sim$ $\exp \left[-\left(E_{2}\left(t_{i}\right)\right)^{2} /(2 \mathcal{E})\right]$ holds exactly.

In the case of system $b$, as shown in Fig. 3, the gap between the mean trajectories in the mixed and forward picture is in the range expected for the values of the 


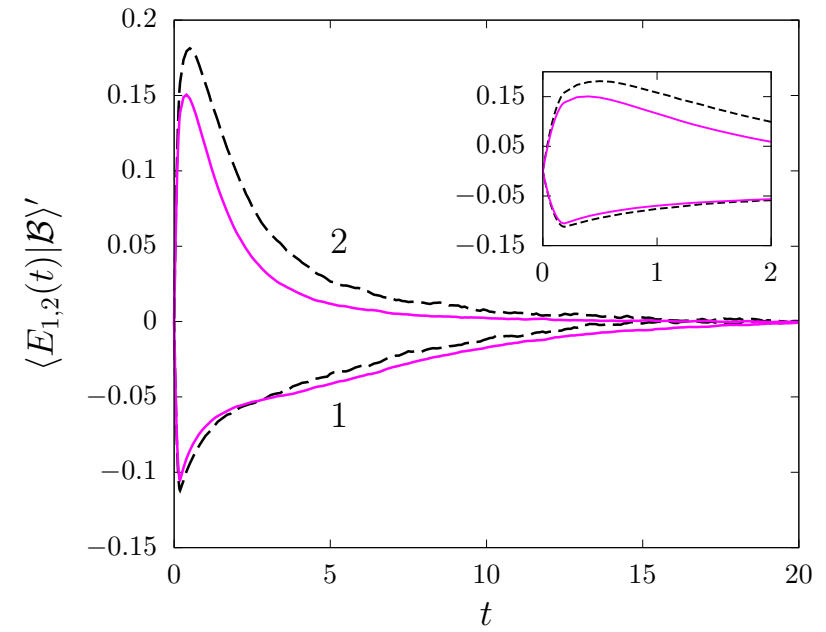

FIG. 3. Mean trajectory deviation from the decoupled limit in the case of system $b$. Mixed picture black dashed line; forward picture light continuous line (magenta online).

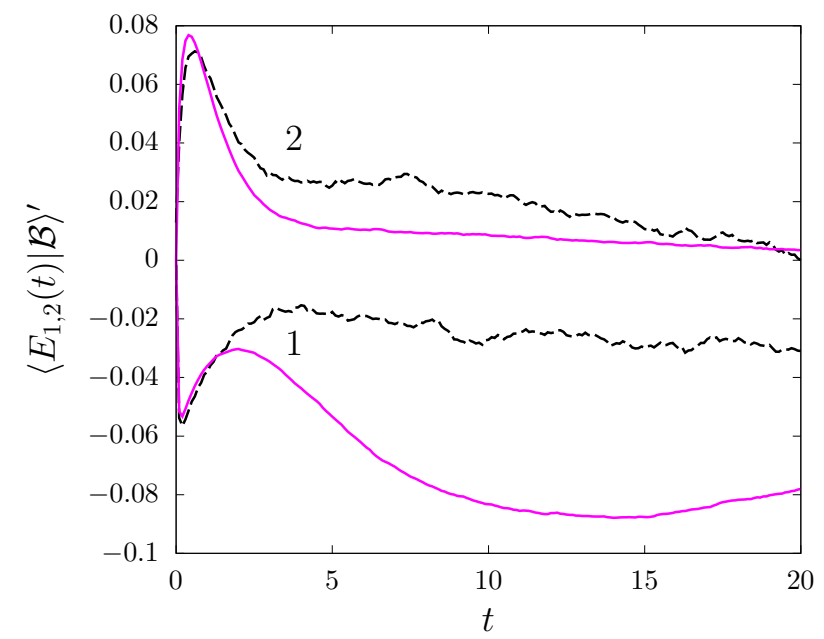

FIG. 4. Same as Fig. 3 in the case of system $c$.

coupling $g$ considered.

The performance of the mixed approach in the case of system $c$ is much worse. As shown in Fig. 4, the mixed approach heavily underestimates $\left\langle E_{1}(t) \mid \mathcal{B}_{1}\right\rangle^{\prime}$, which is the contribution to the relaxation of $E_{1}$ to the bottom of the potential well at $E_{1}=0$ from interaction with $E_{2}$. The poor performance of the mixed approach appears to be a a manifestation of the breakdown of the perturbative ansatz $g F_{2}^{(1)} \ll F_{2}^{(0)}$ near the potential barrier at $E_{2} \approx 1.8$ (see Fig. 10), where the contribution from $F_{1}^{(1)}$ to the escape of $E_{1}$ from the shallow well to the right is dominant. The situation is similar to the breakdown of the WKB expansion in the vicinity of turning points [35]. In the present case, the poor performance of the mixed backward-forward approach is associated with the long permanence of $E_{1}$ in the shallow potential well to

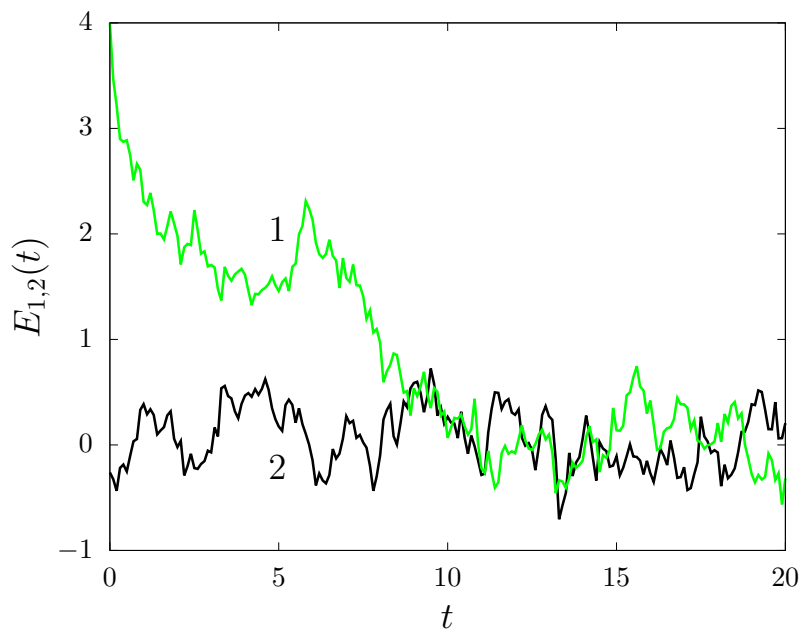

FIG. 5. Individual trajectories for system $c$, obtained from integrating in the forward approach ( $E_{1}$ green online). Notice the long time spent by the system at $E_{1} \gtrsim 2$, before making transition to the potential well at $E_{1} \approx 0$.

the right (see Fig. 5), where the dynamics are dominated by the noise and by the interaction with $E_{2}$.

\section{CONCLUSION}

We have derived a mixed backward-forward approach for the treatment of stochastic systems, with initial and final conditions imposed on different subsets of the variables that describe the dynamics. The main results of the paper are contained in Eqs. (25,26) and (46), which provide the form of the SDE's, together with the procedure for the calculation of conditional averages in our approach. The interaction between forward and backward variables is taken into account by the introduction of additional internal degrees of freedom, which make the dynamics intrinsically nonlocal in time.

Nonlocality in time turns the boundary conditions on the system into statistical constraints in an infinite time domain; this however is precisely what happens, independently of the description, in an incomplete stochastic bridge: by construction, variables in an incomplete stochastic bridge that do not satisfy boundary conditions at an end of the bridge, bring information from the outside into the bridge. It is thus not too surprising that the most natural description of a system in which initial and final conditions are imposed on different sets of variables, is nonlocal in time.

As regards the cosmological models in [25], the implication is that the hypothetical presence of regions in the universe with an arrow of time opposite to ours would require some form of time nonlocality in the interaction between us and "them".

We have derived the mixed backward-forward approach in the case of reversible dynamics described in 
the forward picture by an SDE with additive noise. No additional hypotheses have been made on the form of the SDE's, except the smallness of the interaction between forward and backward variables. For the sake of clarity the derivation has been carried out in the simplest possible case of a two-variable system; the generalization to systems with a higher number of variables, however, is straightforward. A question that remains open is the possible extension of the perturbative expansion to higher orders in the coupling strength. It likewise remains open the question of a possible extension of the approach to the case of irreversible dynamics.

From the point of view of the Monte Carlo evaluation of statistical quantities, the main advantage of the approach is the possibility of treating final conditions as if they were initial conditions in a forward approach. Therefore, as opposed to shooting algorithms, the approach does not need large samples to treat low probability final states; at the same time, it does not have the problems of slow convergence and difficult handling of final points of transition path sampling algorithms [18, 36]. The price is the increased memory requirement implicitas discussed in Sec. V - in a forward-backward description of the dynamics, and the constraint of weak interaction between components of the system with opposite conditioning.

Numerical tests in the simplest possible case of a system with two degrees of freedom show that the mixed backward-forward approach works as long as there is no crossover in the strength of the interaction relative to the unperturbed dynamics. A situation of physical interest where the condition is violated is the crossing of a potential barrier. The situation is similar to that of the WKB method near a turning point, and the procedure to tackle the problem is expected to be the same, namely, to shift to an alternative description in the vicinity of the barrier. How to carry out the procedure, however, remains unclear at the moment, and the matter deserves further study.

\section{Acknowledgements}

Part of this research was carried out at the Mathematics Department of the University of Helsinki. The author thanks Prof. Paolo Muratore Ginanneschi for hospitality. This research was supported in part by the AtMath Collaboration at the University of Helsinki and by FP7 EU project ICE-ARC (Grant agreement No. 603887).

\section{Appendix A: Calculation of the Jacobian determinant}

Consider initially a finite time domain $\left[n_{i}, n_{f}\right]$. We can dispose the entries of the four-index matrix $\partial \Delta_{ \pm} W_{j}(n) / \partial E_{k}(m)$ along the rows and columns of a staggered $2\left(n_{f}-n_{i}-1\right) \times 2\left(n_{f}-n_{i}-1\right)$ matrix
$\mathcal{J}_{\alpha(j, n), \beta(k, m)}$, where indices $\alpha=1,2, \ldots$ map to

$$
\begin{aligned}
(j, n)= & \left(1, n_{i}\right),\left(2, n_{f}\right),\left(1, n_{i}+1\right),\left(2, n_{f}-1\right), \\
& \ldots,\left(1, n_{f}-1\right),\left(2, n_{i}+1\right)
\end{aligned}
$$

and indices $\beta=1,2, \ldots$ map to

$$
\begin{aligned}
(k, m)= & \left(1, n_{i}+1\right),\left(2, n_{f}-1\right),\left(1, n_{i}+2\right),\left(2, n_{f}-2\right), \\
& \ldots,\left(1, n_{f}\right),\left(2, n_{i}\right) .
\end{aligned}
$$

Let us decompose

$$
\mathcal{J}=\overline{\mathcal{J}}+g \tilde{\mathcal{J}}=\overline{\mathcal{J}}[1+g \hat{\mathcal{J}}] .
$$

We see from Eq. (8) that to lowest order in $\Delta t \overline{\mathcal{J}}$ is a banded matrix

$$
\overline{\mathcal{J}}_{\alpha \beta}=\delta_{\alpha \beta}-\delta_{\alpha, \beta+2}+O(\Delta t),
$$

with determinant

$$
\operatorname{det} \overline{\mathcal{J}}=1+O(\Delta t)
$$

and inverse, the lower triangular matrix

$$
\overline{\mathcal{J}}^{-1}=\left[\begin{array}{ccccccc}
1 & 0 & & & & & \\
0 & 1 & 0 & & & & \\
1 & 0 & 1 & 0 & & & \\
0 & 1 & 0 & 1 & 0 & & \\
1 & 0 & 1 & 0 & 1 & 0 & \\
& & & \vdots & & &
\end{array}\right]+O(\Delta t)
$$

Following standard practice 37], we write the Jacobian $J=|\operatorname{det} \mathcal{J}|$ as a Taylor series:

$$
\begin{aligned}
J & =|\operatorname{det} \overline{\mathcal{J}}||\operatorname{det}(1+g \hat{\mathcal{J}})|=\exp [\operatorname{tr} \ln (1+g \hat{\mathcal{J}})] \\
& =\exp (1+g \operatorname{tr} \hat{\mathcal{J}})+O\left(g^{2}\right),
\end{aligned}
$$

where we have exploited Eq. A5 , and we have disregarded $O(\Delta t)$ terms, that vanish in the continuous limit. Substituting Eqs. (A3) and (A6) into Eq. (A7), we get

$$
\begin{aligned}
\operatorname{tr} \hat{\mathcal{J}} & =\sum_{\alpha \geq 1} \sum_{\beta \geq \alpha}\left[\tilde{\mathcal{J}}_{2 \alpha-1,2 \beta-1}+\tilde{\mathcal{J}}_{2 \alpha, 2 \beta}\right] \\
& =\sum_{m}\left[\sum_{n>m} \frac{\partial \Delta W_{1}\left(t_{n}\right)}{\partial E_{1}\left(t_{m}\right)}+\sum_{n<m} \frac{\partial \Delta W_{2}\left(t_{n}\right)}{\delta E_{2}\left(t_{m}\right)}\right] \\
& =\Delta t \sum_{m}\left[\sum_{n>m} \frac{\partial M_{1}^{(1)}(n)}{\partial E_{1}(m)}-\sum_{n<m} \frac{\partial M_{2}^{(1)}(n)}{\partial E_{1}(m)}\right] \\
& +O(g) .
\end{aligned}
$$

Note that there is no contribution to $\operatorname{tr} \hat{\mathcal{J}}$ from $M_{j}^{(0)}$, which is consequence of the fact that there is no contribution in the sums in Eq. (A8) from $n=m$. This in turn is consequence of the fact that $M_{1}^{(0)}(n)$ does not depend on $E_{1}(n+1)$, and that $M_{2}^{(0)}$ does not depend on and $E_{2}(n-1)$. Taking in Eq. A8 the two limits $\Delta t \rightarrow 0$ and $\left[t_{i}, t_{f}\right] \rightarrow[-\infty, \infty]$ finally yields Eqs. (14) and (15). 


\section{Appendix B: Prescription issues in the mixed picture}

Let us analyze separately the contributions to Eq. (20) from $\mathcal{L}_{P}, \mathcal{L}_{I}$ and $\mathcal{L}_{J}$. We promptly verify that the first term on the right hand side (RHS) of Eq. (23) is a Stratonovich differential [30], which brings no Itô correction when integrating by parts, and that the second term is proportional to the noise cross correlation, which is $O(g)$. Hence, to the order considered, we can set $\mathcal{L}_{P}=0$.

It is difficult to prove in general that $\mathcal{L}_{I}=\mathcal{L}_{J}=0$, but we can verify a posteriori that the condition is satisfied in the case of Eqs. (30+31).

In the case of $\mathcal{L}_{I}$, the fact that the argument $\mathbf{E}$ in the functionals $\hat{M}_{j}[\mathbf{E}, t]$, as illustrated in Eqs. 30,31), depends on time only through a dummy integration variable, guarantees that $\dot{\hat{M}}_{j}[\mathbf{E}, t]=\partial_{t} \hat{M}_{j}[\mathbf{E}, t]$. Hence, substituting $V_{j}=\hat{M}_{j}$ in Eq. (18) does not generate an Itô correction, and therefore $\mathcal{L}_{I}=0$.

As regards $\mathcal{L}_{J}$, Eqs. (30-31) tell us that $\hat{M}_{1}(\tau)$ and $\hat{M}_{2}(\tau)$ receive contribution from $\mathbf{E}(t>\tau)$ in one case, from $\mathbf{E}(t<\tau)$ in the other. In addition to this, we have seen by working in discrete time, that $M_{j}^{(1)}(n)$ depends as a function on $E_{j}(m)$ only for $m=n$ [see Eqs. (21) and (22)]. However, from Eqs. (15) and (A8), $\mathcal{L} \neq 0$ only if $M_{1}^{(1)}(n)$ depends on $E_{1}(m<n)$, or $M_{2}^{(1)}(n)$ depends on $E_{2}(m>n)$, or both. Hence, $\mathcal{L}_{J}=0$.

\section{Appendix C: Correction terms in the evaluation of conditional averages}

We evaluate the averages in Eqs. (41,43) in terms of the PDF

$$
\rho[\overline{\mathbf{E}}]=\rho\left(\bar{E}_{1}\left(t_{i}\right), \bar{E}_{2}\left(t_{f}\right)\right) \rho\left[\overline{\mathbf{E}} \mid \bar{E}_{1}\left(t_{i}\right), \bar{E}_{2}\left(t_{f}\right)\right] .
$$

We start by evaluating the PDF $\rho(\mathcal{B})$ in Eq. (38). We Taylor expand $\mathcal{B}$ around $\overline{\mathbf{E}}$ and substitute into $\left\langle\delta_{\mathcal{B}}\right\rangle=$ $\int[\mathrm{d} \bar{E}] \rho[\overline{\mathbf{E}}] \delta_{\mathcal{B}}$. Equation (C1) gives us then

$$
\begin{aligned}
\rho(\mathcal{B}) & =\rho(\overline{\mathcal{B}})\left\{1-g\left[\nabla_{i f} \cdot\left\langle\tilde{\mathbf{E}}_{i f} \mid \overline{\mathcal{B}}\right\rangle\right.\right. \\
& \left.\left.+\left\langle\tilde{\mathbf{E}}_{i f} \mid \overline{\mathcal{B}}\right\rangle \cdot \nabla_{i f} \ln \rho(\overline{\mathcal{B}})\right]\right\}+O\left(g^{2}\right),
\end{aligned}
$$

where

$$
\nabla_{i f}=\left(\partial_{E_{1}^{i}}, \partial_{E_{2}^{f}}\right), \quad \tilde{\mathbf{E}}_{i f}=\left(\tilde{E}_{1}\left(t_{i}\right), \tilde{E}_{2}\left(t_{f}\right)\right)
$$

In Eq. (C2) we can approximate $\rho(\overline{\mathcal{B}}) \simeq \rho^{(0)}(\overline{\mathcal{B}})=$ $\rho^{(0)}\left(\overline{\mathcal{B}}_{1}\right) \rho^{(0)}\left(\overline{\mathcal{B}}_{2}\right)$, where, thanks to reversibility of the dynamics,

$$
\rho^{(0)}\left(\overline{\mathcal{B}}_{1}\right) \sim \exp \left\{-\frac{1}{\mathcal{E}} \int^{E_{1}^{i}} \mathrm{~d} \bar{E}_{1} F_{1}^{(0)}\left(\bar{E}_{1}\right)\right\}
$$

and

$$
\rho^{(0)}\left(\overline{\mathcal{B}}_{2}\right) \sim \exp \left\{-\frac{1}{\mathcal{E}} \int^{E_{2}^{f}} \mathrm{~d} \bar{E}_{2} F_{2}^{(0)}\left(\bar{E}_{2}\right)\right\} .
$$

By putting together Eqs. (38) and (42) with Eqs. (C2) and (C4) 5 , we then get

$$
\frac{\mathcal{N}}{\overline{\mathcal{N}}}-1=g\left[\nabla_{i f}-\frac{\mathbf{F}_{i f}^{(0)}}{\mathcal{E}}\right] \cdot\left\langle\tilde{\mathbf{E}}_{i f} \mid \overline{\mathcal{B}}\right\rangle+O\left(g^{2}\right)
$$

where

$$
\mathbf{F}_{i f}^{(0)}=\left(F_{1}^{(0)}\left(E_{1}^{i}\right), F_{2}^{(0)}\left(E_{2}^{f}\right)\right) .
$$

We can repeat the procedure with the second line of Eq. (43), to obtain

$$
\begin{aligned}
\tilde{Z}[\mathbf{J}] & =\left[\frac{\mathbf{F}_{i f}^{(0)}}{\mathcal{E}}-\nabla_{i f}\right] \cdot\left\langle\tilde{\mathbf{E}}_{i f} \exp \left[\mathrm{i} \int \mathrm{d} t \mathbf{J}(t) \cdot \overline{\mathbf{E}}(t)\right] \mid \overline{\mathcal{B}}\right\rangle \\
& -\left\langle\exp \left[\mathrm{i} \int \mathrm{d} t \mathbf{J}(t) \cdot \overline{\mathbf{E}}(t)\right] \mid \overline{\mathcal{B}}\right\rangle \\
& \times\left[\frac{\mathbf{F}_{i f}^{(0)}}{\mathcal{E}}-\nabla_{i f}\right] \cdot\left\langle\tilde{\mathbf{E}}_{i f} \mid \overline{\mathcal{B}}\right\rangle,
\end{aligned}
$$

and from here we get Eq. 451.
[1] M. L. Puterman, Markov decision processes: discrete stochastic dynamic programming (John Wiley \& Sons, New York, 1994).

[2] D. J. Evans and D. J. Searles, Causality, response theory and the second law of thermodynamics, Phys. Rev. E 53, 5808 (1996).

[3] R. Van Handel, Stochastic calculus, filtering, and stochastic control, Lecture notes in Physics (Springer, Berlin, 2007).

[4] D. Nualart, Noncausal stochastic integrals and calculus, in Stochastic analysis and related topics (Springer,
Berlin, 1988) pp. 80-129.

[5] B. Øksendal, An introduction to Malliavin calculus with applications to economics, Lecture notes in Physics (Springer, Berlin, 1996).

[6] E. Schrödinger, Über die Umkehrung der Naturgesetze, Sitzber. Preuß. Akad. Wiss. Phys.-Math K41, 144 (1931).

[7] P. Glasserman, Monte Carlo Methods in Financial Engineering (Springer, Berlin, 2011).

[8] J. S. Horne, E. O. Garton, S. M. Krone, and J. S. Lewis, Analyzing animal movements using Brownian bridges, 
Ecology 89, 2354 (2007).

[9] M. Glanzer and G.-C. Pflug, Multiscale stochastic optimization: modeling aspects and scenario generation, Comput. Optim. Appl. 75, 1 (2000).

[10] P. D. Drummond, Forward, backward, and weighed stochastic bridges, Phys. Rev. E 96, 042123 (2017).

[11] E. Pardoux and S. Peng, Adapted solution of a backward stochastic differential equation, Systems Control Lett. 14, 55 (1990).

[12] J. L. Doob, Conditional Brownian motion and the boundary limits of harmonic functions, Bull. Soc. Math. France 85, 431 (1957).

[13] H. Orland, Generating transition paths by Langevin bridges, J. Chem. Phys. 134, 174114 (2011).

[14] P. E. Kloeden and E. Platen, Numerical solutions of stochastic differential equations (Springer, Berlin, 1992) Chap. 16.

[15] P. Glasserman, P. Heidelberg, and P. Shahabuuddin, Variance reduction techniques for estimating value-atrisk, Management Sci. 46, 1349 (2000).

[16] L. Rey Bellet and K. Spilioupulos, Irreversible Langevin samplers and variance reduction: a large deviations approach, Nonlinearity 28, 2081 (2015).

[17] C. Dellago, P. G. Bolhuis, and D. Chandler, Efficient transition path sampling: Application to Lennard-Jones cluster rearrangements, J. Chem. Phys. 108, 9236 (1998).

[18] G. E. Crooks and D. Chandler, Efficient transition path sampling for nonequilibrium stochastic dynamics, Phys. Rev. E 64, 026109 (2001).

[19] R. Chetrite and H. Touchette, Nonequilibrium markov processes conditioned on large deviations, in Annales Henri Poincaré, Vol. 16 (Springer, 2015) pp. 2005-2057.

[20] B. Derrida and T. Sadhu, Large deviations conditioned on large deviations i: Markov chain and Langevin equation, J. Stat. Phys. 176, 773 (2019).

[21] A. S. J. S. Mey, P. L. Geissler, and J. P. Garrahan, Rareevent trajectory ensemble analysis reveals metastable dynamical phases in lattice proteins, Phy. Rev. E 89, 032109 (2014).
[22] M. Delarue, P. Koehl, and H. Orland, Ab initio sampling of transition paths by conditioned Langevin dynamics, J. Chem. Phys. 147, 152703 (2017).

[23] M. I. Dykman, E. Mori, J. Ross, and P. Hunt, Large fluctuations and optimal paths in chemical kinetics, J. Chem. Phys. 100, 5735 (1994).

[24] J. Laurie and F. Bouchet, Computation of rare transitions in the barotropic quasi-geostrophic equations, New Journal of Physics 17, 015009 (2015).

[25] L. S. Schulman, Opposite thermodynamic arrows of time, Phys. Rev. Lett. 83, 5419 (1999).

[26] C. Jarzynski and D. K. Wójcik, Classical and quantum fluctuation theorems for heat exchange, Phys. Rev. Lett. 92, 230602 (2004).

[27] S. Ciliberto, A. Imparato, A. Naert, and M. Tanase, Heat flux and entropy produced by thermal fluctuations, Phys. Rev. Lett. 110, 180601 (2013).

[28] D. V. Averin and J. P. Pekola, Violation of the fluctuation-dissipation theorem in time-dependent mesoscopic heat transport, Phys. Rev. Lett. 104, 220601 (2010).

[29] D. S. Golubev and J. P. Pekola, Statistics of heat exchange between two resistors, Phys. Rev. B 92, 085412 (2015).

[30] H. Risken, The Fokker-Planck Equation (Springer, Berlin, 1996).

[31] L. Onsager and S. Machlup, Fluctuations and irreversible processes, Phys. Rev. 91, 1505 (1953).

[32] R. Graham, Path integral formulation of general diffusion processes, Z. Phys. B 26, 281 (1977).

[33] M. Dykman and M. Krivoglaz, Theory of fluctuational transitions between stable states of a nonlinear oscillator, Sov. Phys. JETP 50, 30 (1979).

[34] P. C. Martin, E. Siggia, and H. Rose, Statistical dynamics of classical systems, Phys. Rev. A 8, 423 (1973).

[35] L. D. Landau and E. M. Lifshitz, Quantum mechanics: non-relativistic theory (Elsevier, 2013).

[36] D. M. Ceperley, Path integrals in the theory of condensed helium, Rev. Mod. Phys. 67, 279 (1995).

[37] J. Zinn-Justin, Quantum field theory and critical phenomena (Clarendon Press, Oxford, 2002) Chap. 4. 